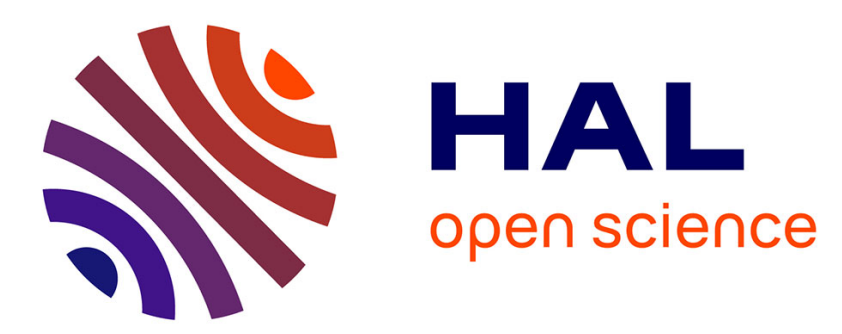

\title{
Semiempirical calculations on the electronic properties of finite-length models of carbon nanotubes based on Clar sextet theory
}

Francesco Mercuri

\section{- To cite this version:}

Francesco Mercuri. Semiempirical calculations on the electronic properties of finite-length models of carbon nanotubes based on Clar sextet theory. Molecular Simulation, 2008, 34 (10-15), pp.905-908. 10.1080/08927020802101726 . hal-00515032

\section{HAL Id: hal-00515032 \\ https://hal.science/hal-00515032}

Submitted on 4 Sep 2010

HAL is a multi-disciplinary open access archive for the deposit and dissemination of scientific research documents, whether they are published or not. The documents may come from teaching and research institutions in France or abroad, or from public or private research centers.
L'archive ouverte pluridisciplinaire HAL, est destinée au dépôt et à la diffusion de documents scientifiques de niveau recherche, publiés ou non, émanant des établissements d'enseignement et de recherche français ou étrangers, des laboratoires publics ou privés. 


\section{Molecular Simulation \\ Journal of \\ Experimental Nanoscience \\ $\because$ Taylor \& Francis \\ Taglor \& Francis Group}

\section{Semiempirical calculations on the electronic properties of finite-length models of carbon nanotubes based on Clar sextet theory}

\begin{tabular}{|r|l|}
\hline Journal: & Molecular Simulation/Journal of Experimental Nanoscience \\
\hline Manuscript ID: & GMOS-2008-0013.R1 \\
\hline Journal: & Molecular Simulation \\
\hline $\begin{array}{r}\text { Date Submitted by the } \\
\text { Author: }\end{array}$ & 04-Mar-2008 \\
\hline Complete List of Authors: & $\begin{array}{l}\text { Mercuri, Francesco; ISTM-CNR, Department of Chemistry, } \\
\text { University of Perugia }\end{array}$ \\
\hline Keywords: & $\begin{array}{l}\text { Materials Studio, carbon nanotubes, Clar sextet theory, electronic } \\
\text { structure, semiempirical methods }\end{array}$ \\
\hline \multicolumn{2}{|l}{} \\
\hline
\end{tabular}

\section{SCHOLARONE" Manuscripts}


Molecular Simulation

Vol. X, No. X, Month 200X, 000-000

\title{
RESEARCH ARTICLE
}

\author{
Semiempirical calculations on the electronic properties of finite-length models of carbon nanotubes \\ based on Clar sextet theory
}

Materials Studio Special Issue

\author{
Francesco Mercuri
}

ISTM-CNR and Department of Chemistry, University of Perugia, Italy

\begin{abstract}
Electronic structure calculations on carbon nanotubes (CNTs) and related materials constitute an active and challenging field of research. Computational approaches to the problem require i) the definition of consistent models of CNTs ii) calculation of the properties on such models with accurate electronic structure methods. In this work, we perform semiempirical AM1 calculations on finite-length models of CNTs based on Clar sextet theory. In particular, the use of the Accelrys Materials Studio package allows to perform both the model building and computing steps through a simple and user-friendly interface. The consistency of such an approach are demonstrated by the smooth and monotonic decrease of the highest-occupied molecular orbital (HOMO) - lowestunoccupied molecular orbital (LUMO) energy gap with length of models for metallic nanotubes and fast convergence to a finite value for models of semiconducting CNTs. As a further example of the applicability of the method outlined in this paper, the dependence of the HOMO-LUMO gap with the diameter for a series of analogous CNTs was also analysed.
\end{abstract}

Keywords: Materials Studio; carbon nanotubes; Clar sextet theory; electronic structure; semiempirical methods

\footnotetext{
*Email: merc@ thch.unipg.it
} 


\section{Introduction}

The past few years have witnessed an impressive boost of research disciplines related to nanotechnology. In particular, the properties of carbon nanotubes (CNTs) have been deeply investigated [1] in view of their potential use in several applications of nanotechnology [2,3] or in nanostructured devices [4]. Consequently, CNTs and related materials have been the subject of comprehensive research work, in which their peculiar properties have been analysed in detail. Beside experimental studies, computer simulations have also been spotted as successful investigation techniques in the study of nanostructured materials. In particular, electronic structure methods, such as density functional theory (DFT) or semi-empirical techniques represent a preferential tool for numerical simulations, being able to provide accurate results for systems constituted of up to a few hundreds of atoms. However, similarly to other nanostructured systems, theoretical investigations on CNTs rely on an accurate definition of models to be employed in calculations. Such models should be ideally constituted by a small number of atoms and should be also representative of the system under study.

Computational investigations on single-walled CNTs are usually performed on either periodic or finite models of the hexagonal carbon atom network. On the one hand, periodic models are generally accurate and account properly for the properties of a virtually infinite quasi-unidimensional system. On the other hand, calculations on periodic systems are computational demanding, especially when the periodic unit cell is constituted by hundreds of atoms, as in the case of chiral CNTs. An alternative is based on the use of finite-length models, as used in previous work [5-7]. However, the representativity of finite-length models in describing the properties of nanosized objects is a non-trivial problem, and this also applies to the case of CNTs [8]. In particular, the construction of finite-length models of CNTs should take into account the peculiarities of the electronic structure at the sidewall, concerning especially $\pi$-conjugation and aromaticity.

Recent work, has indicated the use of Clar sextet theory $[9,10]$ as appropriate for the description of the electronic structure of the sidewall of CNTs [11-13], in analogy to the case of polycyclic aromatic hydrocarbons [14]. This approach allowed the construction of finite length models of CNTs which are good representatives of their infinite counterparts. In particular, models defined in terms of finite-length Clar clusters (FLCCs) [15] provide accurate electronic properties, as demonstrated for calculations performed at the DFT level [15], and exhibit several computational advantages. Moreover, methods based on semiempirical potentials and particularly the AM1 method [16] allow to describe correctly the electronic structure and the reactivity of CNTs, as for example demonstrated by Orsmy and King in the study of hydrogenation reactions at the sidewall [12].

In this paper, we make use of the Materials Studio package to build models of zig-zag, armchair and chiral CNTs based on FLCCs and evaluate their structural and electronic properties at the semiempirical AM1 level through the VAMP [17] module of Materials Studio. Our results shows as the FLCC approach can be easily integrated in the Builder module of Materials Studio and demonstrate also the consistency of the electronic properties, calculated at the AM1 level, with the results of previous DFT calculations.

\section{Computational details}

Models of semiconducting zig-zag, armchair and chiral CNTs based on FLCCs were constructed through the Builder module of the Materials Studio package. In particular, as representatives of more general cases, models of $(7,7),(8,0),(6,4)$ and $(6,5)$ CNTs were taken into account. Moreover, the dependence of electronic properties with the nanotube diameter for an analogous set of systems was studied for semiconducting chiral $(n, n-2)$ CNTs, with $n$ ranging from 5 to 9 . Finite-length models of nanotubes were built by starting from the corresponding periodic unit cell, as provided by the Materials Studio Builder, recutting and saturating the edges of the FLCCs with hydrogen atoms as described in Ref. [15]. 
Geometries were optimised at the AM1 level of theory as implemented in the VAMP module[17] of Materials Studio and electronic properties were evaluated at the same level.

\section{Results and discussion}

As discussed in previous work [11,15], Clar sextet theory can be conveniently applied to the hexagonal network of the CNT sidewall by defining two translational basis vectors, which reflect the translational symmetry of a fully benzenoid graphene sheet and are connected to the 2-dimensional $(n, m)$ vector basis of the nanotubes sidewall [18] by algebraic relationships [11]. The Clar vector basis allows the definition of a primitive cell of the CNTs, from which the infinite CNT can be generated by application of a screw axis operation along the nanotube principal axis [15]. Ormsby and King demonstrated [11] that the description of the sidewall in terms of the primitive Clar cell leads to a classification of the CNTs based on the parameter $R$, which is defined as: $R=(n-m, 3)$, where $n$ and $m$ are the nanotube chiral vectors. This classification accounts for the main features of the electronic structure at the sidewall and indicates whether the nanotube is metallic $(R=0)$ or semiconducting $(R=1$ and $R=2)$. The calculations presented in this work were performed on FLCC models of CNTs ranging from 2 to 6 replica of the Clar unit cell.

Figure 1 shows the optimised geometry of FLCC models of $(7,7),(8,0),(6,4)$ and $(6,5)$ CNTs for the case of three Clar cells. Geometries obtained at the AM1 level are in excellent agreement with the geometries evaluated at the DFT level [15], the larger deviations being related to the position of terminal hydrogens. The root mean square deviation (RMSD) between the DFT and AM1 optimised geometries was evaluated by first rotating the structures to minimise the RMSD, according to the method of Kabsch [19], then calculating the RMSD, defined as:

$$
d_{R M S}=\left[\sum_{i=1}^{N}\left\|r_{D F T}-r_{A M 1}\right\|^{2}\right]^{1 / 2}
$$

where $\boldsymbol{r}_{D F T}$ and $\boldsymbol{r}_{A M I}$ are the positions of atoms for structures computed at the DFT and AM1 levels, respectively, and $N$ is the total number of atoms in the structure. As shown in Table 1, RMSDs between DFT (B3LYP/3-21G) and AM1 geometries are below $0.085 \AA$ in all cases considered, thus pointing semiempirical methods as reliable methods for obtaining accurate structures of finite-length models of CNTs.

As indicator of the convergence of the electronic properties with the number of Clar cells, the highestoccupied molecular orbital (HOMO) - lowest unoccupied molecular orbital (LUMO) energy gap was evaluated for FLCC models of CNTs by varying the number of Clar cells replica. As stated previously, finitelength models should be representative of the corresponding infinite nanotubes and, consequently, exhibit a consistent and monotonic convergence of their properties towards the asymptotic limit for an infinite number of units. Figure 2 shows the dependence of the HOMO-LUMO energy gap from the number of Clar cells for FLCC models of the CNTs considered.

For the metallic $(7,7)$ nanotube $(R=0)$, the HOMO-LUMO gap exhibits a regular and monotonic decrease with the model length. This result is consistent with the properties expected for finite-length models of a metal, where the energy gap is zero at infinite length. Moreover, the monotonic trend indicates that FLCC models are well behaved and map properly, even at short lengths, the electronic properties of metallic nanotubes, in agreement with our previous DFT calculations [15]. Inversely, the HOMO-LUMO gap of semiconducting $(8,0),(6,4)(R=2)$ and $(6,5)(R=1)$ nanotubes converge quickly with the number of Clar cells. This indicates as the electronic properties of infinite nanotubes can be conveniently represented by using short-sized FLCC models. The HOMO-LUMO gap of models for $(6,5)$ and $(6,4) \mathrm{CNTs}$ converges within $0.05 \mathrm{eV}$ with 5 Clar cells whereas the model of the $(8,0) \mathrm{CNT}$ exhibit a slightly slower convergence $(0.15 \mathrm{eV}$ for $5 \mathrm{Clar}$ cells $)$.

Although the HOMO-LUMO gap was the only parameter considered in this study to assess the reliability of our computational approach, it must be noted that frontier orbitals are usually good indicator of many 
electronic properties of molecular and complex systems, including reactivity [20]. Therefore, calculations performed at the AM1 level on CNT models based on chemical intuition, as in the case of FLCCs, are expected to provide an accurate description of the electronic structure of nanotubes and, consequently, reliable information on the sidewall reactivity [12]. As a further confirmation, we also checked the convergence of the dipole moment of CNTs, calculated at the AM1 level, with the length of the corresponding FLCC model. As shown in Figure 4 for the examples of the $(8,0)$ and $(6,5)$ CNTs, total dipole moments of FLCC models converge promptly to a finite value, with an accuracy of about 0.02 Debyes for 5 Clar cells.

As stated above, semiempirical AM1 calculations were also performed on models of $(n, n-2)(n=5,6,7,8,9)$ CNTs to assess the effect of the nanotube diameter on the electronic properties for a set of analogous systems. In this case, the HOMO-LUMO energy gap decreases monotonically (see Figure 3), as expected for rolled graphene sheets of decreasing diameters. This finding is in agreement with previous DFT calculations [15] and confirms the possibility of using FLCC models in the study of electronic properties of CNTs at the semiempirical level.

It is worth noting that approaches to the modelling of CNTs based on the conventional translational unit cell do not guarantee an adequate description of the electronic properties of the sidewall, concerning especially features related to $\pi$-conjugation and aromaticity, even if computed with a sophisticated theoretical method [9]. Inversely, the use of models which fulfil the requirements of the electron conjugation pattern on the sidewall even for short-sized clusters is "additive" by construction and allows to obtain consistent and accurate results even with the application of relatively simple (and inexpensive) simulation techniques.

\section{Conclusions}

In summary, we demonstrated the use of the Materials Studio package as an integrated tool for the construction of finite-length CNT models based on Clar sextet theory and for the evaluation of their properties at the semiempirical AM1 level. Optimised geometries obtained at the AM1 level agree remarkably well with the results of higher-level DFT (B3LYP/3-21G) calculations. Moreover, the electronic properties of CNT models based on FLCCs calculated at the AM1 level are good representatives of their infinite counterparts. In particular, the AM1 HOMO-LUMO energy gap decreases monotonically to zero gap or converges monotonically to a finite value for models of metallic and semiconducting CNTs, respectively, in agreement | with previous studies [15]. Dipole moment calculations also confirm the reliability of the FLCC approach. Furthermore, semiempirical calculations performed on FLCC models allow a correct description of the change of the electronic properties with the diameter for a set of analogous CNTs.

Therefore, the application of user-friendly packages for the definition of computational models of CNTs based on chemical considerations, such as the Material Studio builder, coupled to semiempirical methods, represents a valuable method for obtaining quantitative information on the electronic properties of CNTs at a relative cheap computational cost. The results and the methodology presented in this work can be extended to studies where the electronic properties of CNTs play a crucial role, as in the case of investigations on the reactivity at the sidewall.

References

[1] Y. N. Xia, P. D. Yang, Y.G. Sun, Y. Y. Wu, B. Mayers, B. Gates, Y. D. Yin, F. Kim and Y. Q. Yan, Onedimensional nanostructures: Synthesis, characterization, and applications, Adv. Mater. 15 (2003), pp. 353-389.

[2] R. H. Baughman, A. A. Zakhidov and W. A. de Heer, Carbon nanotubes - the route toward applications, Science 297 (2002), pp. 787-792. 
[3] F. Mercuri and A. Sgamellotti, Theoretical investigations on the functionalization of carbon nanotubes, Inorg. Chimica Acta 360 (2007), pp. 785-793.

[4] S. J. Tans, A. R. M. Verschueren and C. Dekker, Room-temperature transistor based on a singled carbon nanotube, Nature 393 (1998), pp. 49-52.

[5] A. Rochefort, D. R. Salahub and P. Avouris, Effects of finite length on the electronic structure of carbon nanotubes, J. Phys. Chem. B 103 (1999), pp. 641-646.

[6] J. Cioslowski, N. Rao and D. Moncrieff, Electronic structure and energetic of $[5,5]$ and $[9,0]$ single-walled carbon nanotubes, J. Am. Chem. Soc. 124 (2002), pp. 8485-8489.

[7] F. Mercuri and A. Sgamellotti, DFT methods in the chemistry of carbon nanotubes, in Chemistry of carbon nanotubes, V. Basiuk and E. Basiuk eds., American Scientific Publishers, Stevenson Ranch CA, 2007.

[9] H. F. Bettinger, Effects of finite carbon nanotubes length on sidewall addition of fluorine atom and methylene, Org. Lett. 6 (2004), pp. 731-734.

[9] E. Clar, Polycyclic hydrocarbons, Academic Press, New York, 1964.

[10] E. Clar, The aromatic sextet, Wiley, London, 1972.

[11] J. L. Ormsby, and B. T. King, Clar valence bond representation of pi-bonding in carbon nanotubes, J.Org. Chem. 69 (2004), pp. 4287-4291.

[12] J. L. Ormsby, and B. T. King, The regioselectivity of addition to carbon nanotubes segments, J.Org. Chem. 69 (2007), pp. 4025-4038.

[13] Y. Matsuo, K. Tahara and E. Nakamura, Theoretical studies on structures and aromaticity of finite-length armchair carbon nanotubes, Org. Lett. 5 (2003), pp. 3181-3184.

[14] M. Randic, Aromaticity of polycyclic conjugated hydrocarbons, Chem. Rev. 103 (2003), pp. 3449-3605.

[15] M. Baldoni, A. Sgamellotti and F. Mercuri, Finite-length models of carbon nanotubes based on Clar sextet theory, Org. Lett. 9 (2007), pp. 4267-4270.

[16] M. J. S. Dewar, E. G. Zoebisch, E.F. Healy, and J. J. P. Stewart, The development and use of quantummechanical molecular-models .76. AM1 - a new general-purpose quantum-mechanical molecularmodel, J. Am. Chem. Soc. 107 (1985), pp. 3902-3909.

[17] Materials studio v.4.2, VAMP module, Accelrys Inc., San Diego, CA 92121, USA.

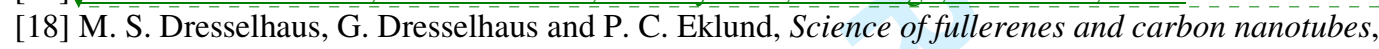
Academic Press, San Diego, 1996.

[19] W. Kabsch, Solution for best rotation to relate 2 sets of vectors, Acta Cryst. A 32 (1976), pp. 922-923.

[20] K. Fukui, Role of frontier orbitals in chemical reactions, Science 218 (1982), pp. 747-754.

Deleted: G. Rauhut, A. Alex, J.

Chandrasekhar, T. Steinke, W. Sauer, B Beck, M. Hutter, P. Gedeck and T. Clark, VAMP 6.5, Oxford Molecular Ltd., The Medawar Centre, Oxford Science Park, Oxford OX4 4GA, England, 1997.

Figure Captions

Figure 1. Optimised geometries for CNT models constituted of 4 Clar cells: a) $(7,7)$; b) $(8,0)$; c) $(6,4)$; d) $(6,5)$.

Figure 2. HOMO-LUMO energy gap of CNT models with respect to the number of Clar cells: a) $(7,7)$; b) $(8,0)$; c) $(6,4)$; d) $(6,5)$.

Figure 3. Variation of the HOMO-LUMO energy gap with the diameter for models of $(n, n-2)$ CNTs $(R=2)$.

Figure 4. Dipole moment of models of $(8,0)$ and $(6,5)$ CNTs with respect to the number of Clar cells.

Table Captions 
1

2

Table 1. RMSD, in Angstroms, between optimized geometries of CNT models calculated at the DFT and AM1 levels, respectively.

\begin{tabular}{cccccc}
\hline & 2 & 3 & 4 & 5 & 6 \\
\hline$(7,7)$ & 0.048 & 0.039 & 0.036 & 0.034 & 0.034 \\
$(8,0)$ & 0.084 & 0.066 & 0.058 & 0.053 & 0.049 \\
$(6,5)$ & 0.064 & 0.050 & 0.046 & 0.049 & 0.045 \\
$(6,4)$ & 0.049 & 0.039 & 0.053 & 0.045 & 0.041 \\
\hline
\end{tabular}

8

9

10

11

12

13

14

15

16

17

18

19

20

21

22

23

24

25

26

27

28

29

30

31

32

33

34

35

36

37

38

39

40

41

42

43

44

45

46

47

48

49

50

51

52

53

54

55

56

57

58

59

60 


\section{Page 7 of 10}

1

2

3

4

5

6

7

8

9

10

11

12

13

14

15

16

17

18

19

20

21

22

23

24

25

26

27

28

29

30

31

32

33

34

35

36

37

38

39

40

41

42

43

44

45

46

47

48

49

50

51

52

53

54

55

56

57

58

59

60 a)

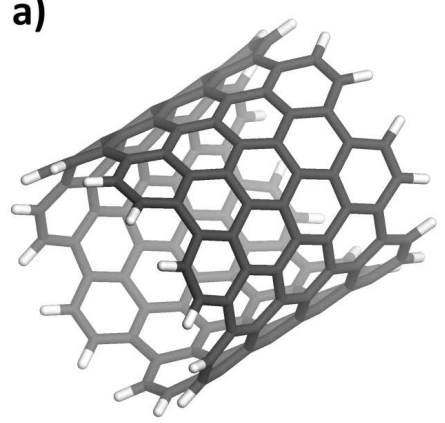

c)

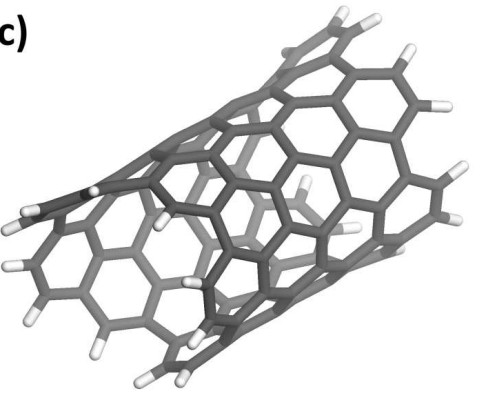

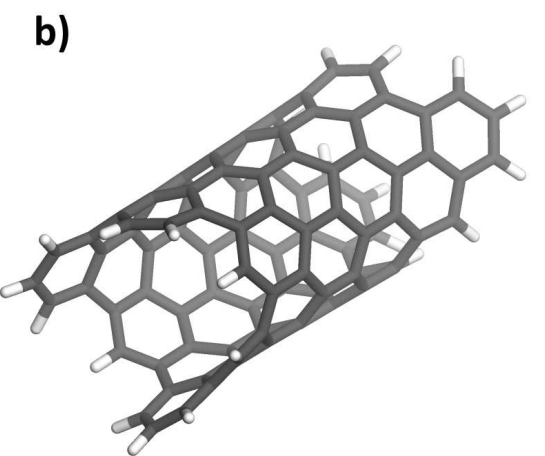

d)

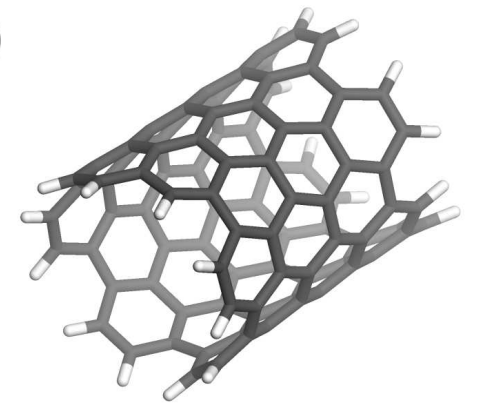

Optimised geometries for CNT models constituted of 4 Clar cells: a) $(7,7)$; b) $(8,0)$; c) $(6,4)$; d) $(6,5)$.

$80 \times 59 \mathrm{~mm}(600 \times 600 \mathrm{DPI})$ 

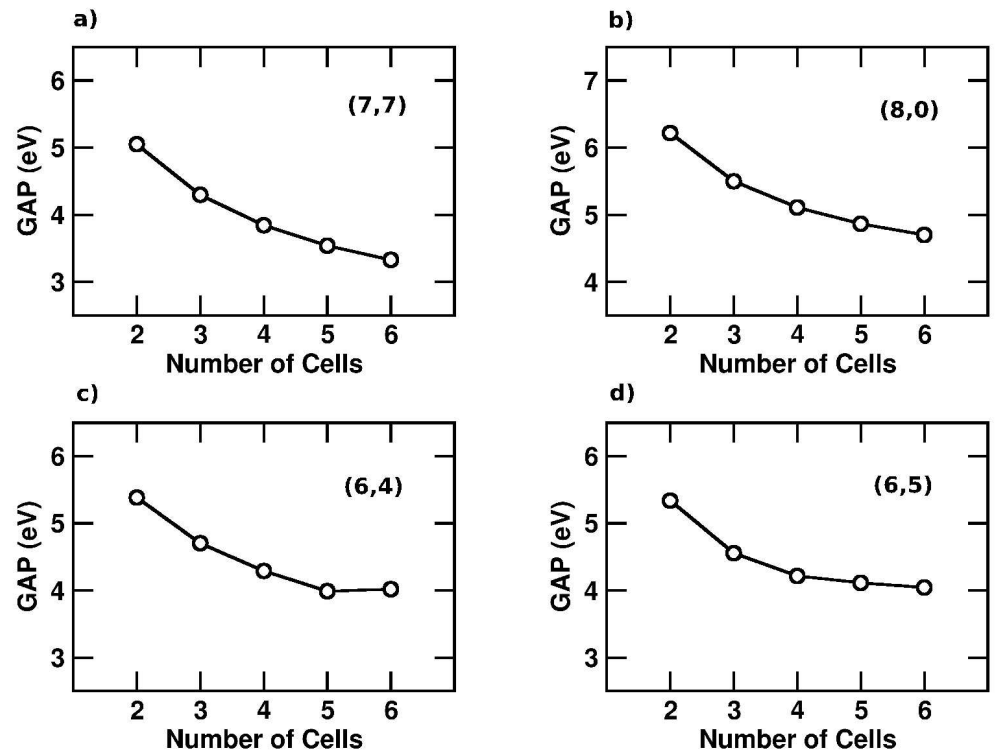

HOMO-LUMO energy gap of CNT models with respect to the number of Clar cells: a) $(7,7) ; b)(8,0)$; c) $(6,4)$; d) $(6,5)$.

$85 \times 60 \mathrm{~mm}(600 \times 600 \mathrm{DPI})$ 


\section{Page 9 of 10}

1

2

3

4

5

6

7

8

9

10

11

12

14

15

16

17

18

19

20

21

22

23

24

25

26

27

28

29

30

31

32

33

34

35

36

37

38

39

40

41

42

43

44

45

46

47

48

49

50

51

52

53

54

55

56

57

58

59

60

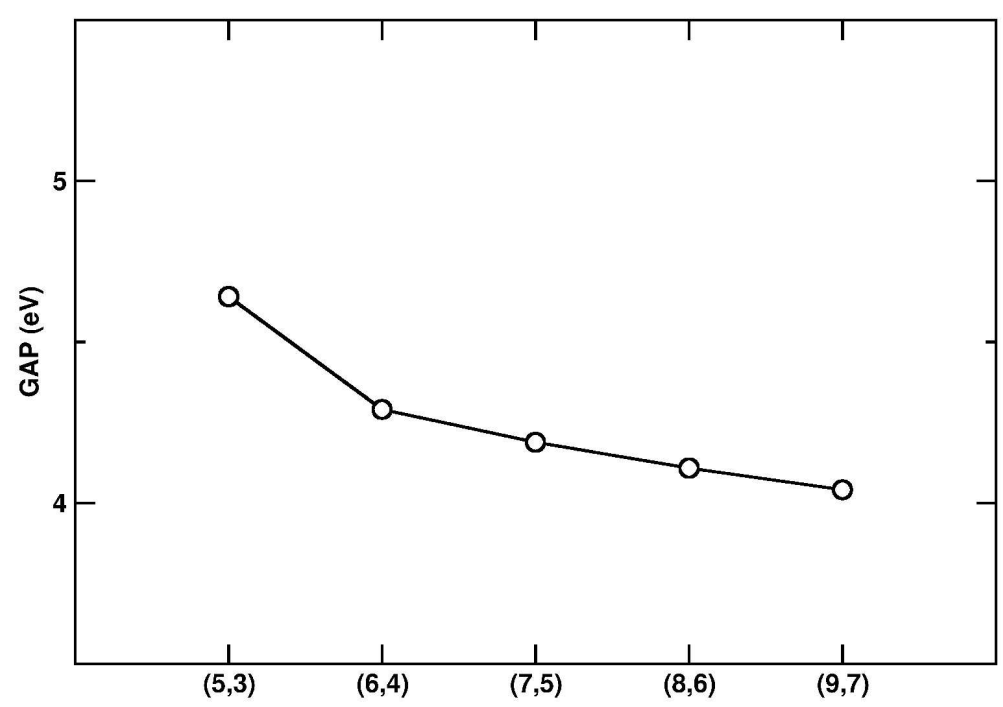

Variation of the HOMO-LUMO energy gap with the diameter for models of $(n, n-2)$ CNTs $(R=2)$. $85 \times 60 \mathrm{~mm}(600 \times 600 \mathrm{DPI})$ 


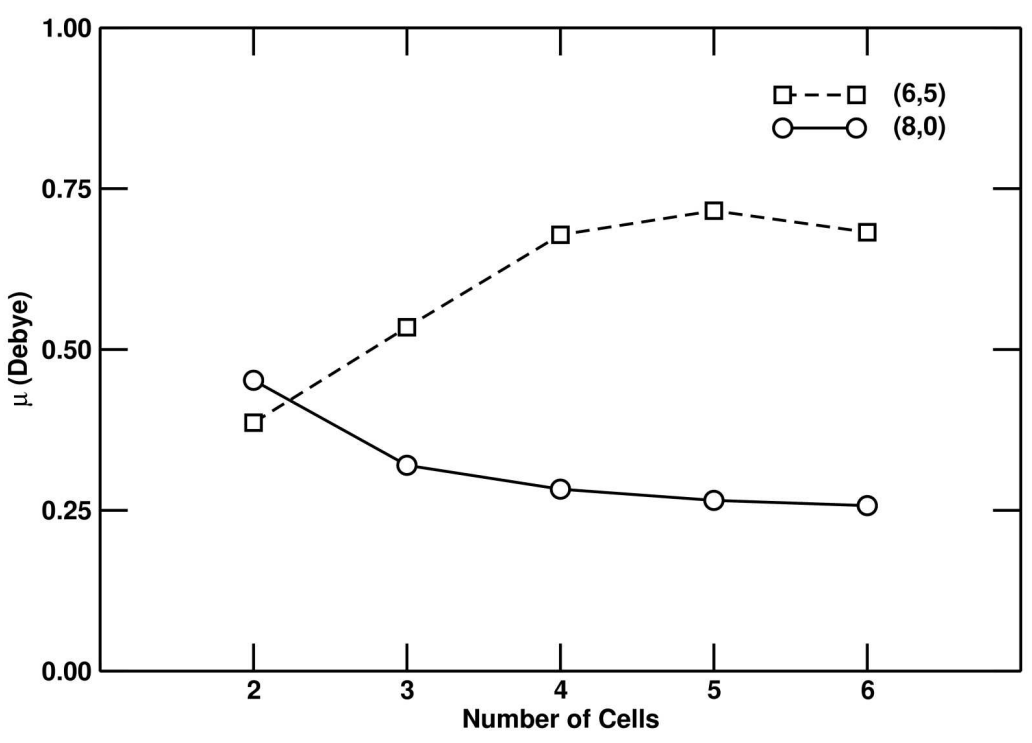

$84 \times 59 \mathrm{~mm}(600 \times 600 \mathrm{DPI})$ 